\title{
Evaluating the Role of Veterinarians in the One Health Approach to Antimicrobial Resistance in Jordan
}

\author{
Randa Bazzi \\ University of Debrecen, Debrecen, Hungary
}

\section{Corresponding Author:}

Randa Bazzi

University of Debrecen

4320 Egytem ter

Debrecen

Hungary

Phone: 36798234650

Email: randaalali12@outlook.com

\section{Abstract}

Background: Antimicrobials, including antibiotics, antivirals, antifungals, and antiparasitics, are drugs used to prevent and treat infections in humans, animals, and plants.

Objective: This study aimed to evaluate the role of knowledge, attitudes, and practices of Jordanian veterinarians in combating antimicrobial resistance (AMR), and to summarize the registered veterinary drugs between 2017 and 2020.

Methods: The descriptive study data were collected using a standardized questionnaire focusing on knowledge, attitudes, and practices of Jordanian veterinarians.

Results: The results were analyzed descriptively and showed that the mean knowledge of the participants who agreed with the statement on AMR definition was 84\%. The majority (95.65\%) agreed that AMR is a challenge for the veterinary sector in Jordan and should be prioritized among other zoonotic diseases. Around $69 \%$ of the participants believe that the misuse and overuse of antimicrobials by quacks - fraudulent and unauthorized practitioners—are the main reasons for AMR challenge. The most common practice among the respondents was recommending clients (farmers, owners, etc) to practice good animal husbandry (80\%). The study also revealed that there was a significant difference $(P=.02)$ between attending training about AMR and their professional sector (private, public, and academic).

Conclusions: This study showed the importance of implementing a continuous education program on antimicrobial resistance to improve veterinarians' knowledge in all aspects of antimicrobial resistance and to increase their advisory skills. Laws should also be enacted to ensure that veterinarians prescribe the correct antimicrobials and improve the surveillance system to monitor the use of antimicrobials in veterinary medicine.

(iproc 2022;8(1):e36375) doi: $10.2196 / 36375$

\section{KEYWORDS}

antimicrobial resistance; veterinarians; knowledge; practices; attitudes; Jordan

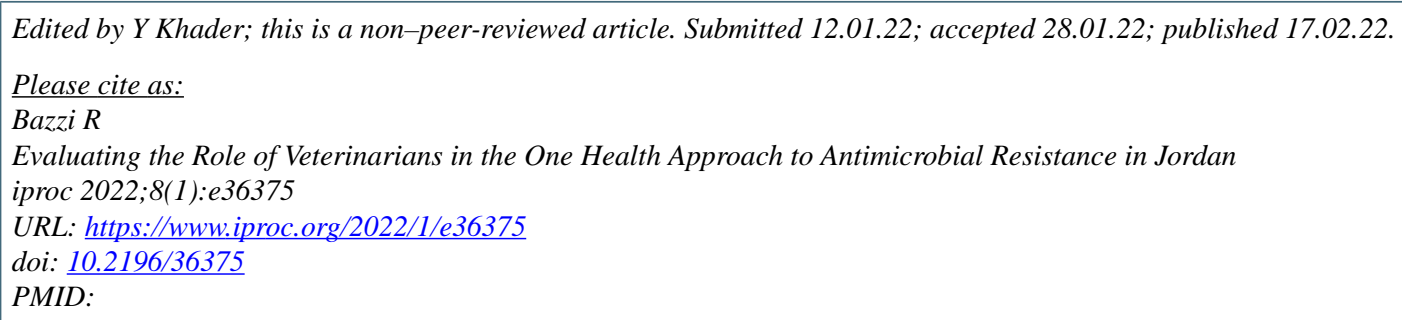


CRanda Bazzi. Originally published in Iproceedings (https://www.iproc.org), 17.02.2022. This is an open-access article distributed under the terms of the Creative Commons Attribution License (https://creativecommons.org/licenses/by/4.0/), which permits unrestricted use, distribution, and reproduction in any medium, provided the original work, first published in Iproceedings, is properly cited. The complete bibliographic information, a link to the original publication on https://www.iproc.org/, as well as this copyright and license information must be included. 